\title{
Bacteriology of Peritonitis in Children Treated at the University Hospital of Marrakech
}

\author{
Taoufik Rokni ${ }^{1}$ *, Adil Rabi ${ }^{1}$, Nabila Soraa ${ }^{1}$, Hassan Ait Bahssain ${ }^{2}$, Younous Said ${ }^{2}$, Tarik Salama ${ }^{2}$, \\ Fouraiji Karima $^{3}$, Kamili El Ouafi El Aouni ${ }^{3}$, Oulad Saiad Mohamed ${ }^{3}$ \\ ${ }^{1}$ Department of Microbiology, Mohammed VI University Hospital, Cadi Ayyad University, Marrakech, Morocco \\ ${ }^{2}$ Department of Pediatric Anesthesia-reanimation, Mohammed VI University Hospital, Cadi Ayyad University, Marrakech, Morocco \\ ${ }^{3}$ Department of Pediatric Surgery, Mohammed VI University Hospital, Cadi Ayyad University, Marrakech, Morocco
}

Email address:

taoufik.rokni@gmail.com (T. Rokni)

${ }^{*}$ Corresponding author

\section{To cite this article:}

Taoufik Rokni, Adil Rabi, Nabila Soraa, Hassan Ait Bahssain, Younous Said, Tarik Salama, Fouraiji Karima, Kamili El Ouafi El Aouni, Oulad Saiad Mohamed. Bacteriology of Peritonitis in Children Treated at the University Hospital of Marrakech. American Journal of Laboratory Medicine. Vol. 4, No. 5, 2019, pp. 87-90. doi: 10.11648/j.ajlm.20190405.12

Received: September 7, 2019; Accepted: September 24, 2019; Published: October 17, 2019

\begin{abstract}
Child peritonitis are severe intra-abdominal infections, involving vital prognosis. The available microbiological data of peritonitis in children are inadequate, and antibiotic therapy is not consensual. Description of the bacteriological profile and the antibiotic resistance of the isolated bacteria in the various samples of peritoneal fluid from the different departments of the University Hospital of Marrakech. It is a descriptive study spread over two years. carried out at the Laboratory of Microbiology of the Mohamed VI Hospital of Marrakech (CHU MED VI), covering all the bacterial strains, isolated in the peritoneal fluid samples from the various pediatric departments of the University Hospital. During this period, 92 samples were treated in the laboratory with a positivity rate of $80 \%$. The average age of his children is 11.7 years with a sex ratio of 1.4 . The infection was polymicrobial in $40 \%$. Escherichia. coli dominated the bacteriological profile of these peritonitis in $74 \%$ of cases, followed by Streptococcus spp (30\%), Pseudomonas aeruginosa (18\%), Enterobacter cloacae (6\%) and Klebsiella pneumoniae (1\%). The susceptibility to amoxicillin in enterobacteria isolated from peritonitis was $32 \%$, $68 \%$ for amoxicillin/clavulanic acid, 92\% for 3rd generation cephalosporins, 97\% for fluoroquinolones, $67 \%$ for cotrimoxazole and $89 \%$ for gentamycin. Only one strain of Pseudomonas aeruginosa was resistant to ceftazidime. All strains remained sensitive to amikacin and carbapenems. Resistance of Enterobacteria to 3rd generation cephalosporins by the production of Extended Spectrum Betalactamase (ESBL) in the isolates was 4\%. This prompts us to reconsider our therapeutic approach. We believe that the association $\mathrm{C} 3 \mathrm{G}+$ aminoglycoside + metronidazole should be used first-line in severe pediatric peritonitis in our context. The quick initiation of an antibiotic therapy adapted to the resistance profile would be an important factor in improving the prognosis, hence the interest of close collaboration between surgeons, anesthesiologist-intensive care and microbiologists.
\end{abstract}

Keywords: Peritonitis, Antibiotherapy, Pediatrics

\section{Introduction}

Child peritonitis are severe intra-abdominal infections, involving vital prognosis. They require both a surgical gesture with emergency antibiotic therapy. This antibiotherapy precedes and completes the surgical procedure, with the aim of controlling bacteremia and the spread of infection [1]. Epidemiological and microbiological data available on community peritonitis of the child are insufficient, and antibiotic therapy does not still the subject of a consensus. On the other hand, in adults, the antibiotic therapy of these intra-abdominal infections was the subject of a French consensus conference recommending the use of amoxicillin-clavulanic acid and aminoglycoside [2]. In the context of emergence of resistant bacterial strains, iterative epidemiological studies are necessary to follow the evolution 
of the resistance of the etiological agents involved in this pathology and to propose adequate therapies [3]. Thus we conducted this work to study the microbiological profile of community peritonitis of the child in our region, to discuss their probabilistic antibiotherapy.

\section{Materials and Methods}

\subsection{Type of Study}

This is a retrospective study carried out at the Laboratory of Microbiology of the Mohamed VI Hospital of Marrakech (CHU MED VI), covering all the bacterial strains, isolated in the peritoneal fluid samples from the various pediatric departments of the University Hospital.

The study was conducted over a 24-month period from January 1, 2017 to December 31, 2018.

\subsection{Inclusion Criteria}

Included in the study, all children hospitalized at the University Hospital of Marrakech who have made a peritoneal sample on an intra-abdominal collection or an echo or scanno-guided puncture of the peritoneal fluid in a postoperative context.

\subsection{Sampling and Patient}

The study focused on peritoneal fluid samples sent for cytobacteriological examination. For each sample received, a quantitative and qualitative cytology was performed with a direct Gram-stained examination to check for the presence of any bacteria. The cultivation of the sampling was done systematically on enriched and selective media.

\subsection{Bacterial Identification and Antibiogram}

Bacterial identification of positive cultures was carried out by conventional methods based on morphological and biochemical characters. The study of susceptibility to different antibiotics was made according to the recommendations of the Committee of the antibiogram of the French society of microbiology CASFM - EUCAST.

An operating chart was used to record the age, sex, clinical, microbiological and evolutionary data of patients.

\section{Results}

During this period, 92 samples were processed in the laboratory. The average age of the children is 11.7 years with a sex ratio $\mathrm{M} / \mathrm{F}$ of 1.4. Four patients were admitted to severe sepsis. The positivity rate was $80 \%(n=74)$ with an isolation of 106 bacterial strains. The infection was poly-microbial in $40 \%$ (Table 1).

Table 1. Distribution of peritonitis according to the number of isolated germs.

\begin{tabular}{lll}
\hline Culture & Nomber & Frequency (\%) \\
\hline Sterile & 18 & 19 \\
Monomicrobial & 37 & 40 \\
Bimicrobial & 31 & 33 \\
Trimicrobial & 6 & 6 \\
Total & 92 & 100 \\
\hline
\end{tabular}

The bacteriological profile of this peritonitis was dominated by enterobacteria with E. coli in $52 \%$ followed by Streptococci in 21\%, and Pseudomonas aeruginosa in $13 \%$ (Table 2).

Table 2. Distribution according to the isolated bacterial species.

\begin{tabular}{|c|c|c|c|c|}
\hline & Family & Germ & Nomber & Frequency \\
\hline \multirow{7}{*}{$\begin{array}{l}\text { GRAM NEGATIVE } \\
\text { BACILLUS }\end{array}$} & \multirow{6}{*}{ ENTEROBACTERIA } & Escherichia coli & 56 & 52 \\
\hline & & Klebsiella pneumoniae & 1 & 1 \\
\hline & & Enterobacter cloacae & 5 & 4 \\
\hline & & Citrobacter freundii & 2 & 2 \\
\hline & & Salmonella Enterica & 1 & 1 \\
\hline & & Shigella boydii & 1 & 1 \\
\hline & PSEUDOMONADACEAE & Pseudomonas aeruginosa & 14 & 13 \\
\hline \multirow[t]{2}{*}{ GRAM POSITIVE COCCI } & STREPTOCOCCUS & Enterocoque spp & 1 & 1 \\
\hline & STAPHYLOCOCCUS & aureus & 2 & 2 \\
\hline TOTALE & & & 106 & 100 \\
\hline
\end{tabular}

The germ associations were represented mainly by Escherichia coli and Streptococcus in 18\% of cases.

Escherichia. coli was 32\% sensitive to amoxicillin, $68 \%$ to amoxicillin-clavulanic acid, $92 \%$ to ceftriaxone and $97 \%$ to fluoroquinolones. All strains remained sensitive to amikacin and imipenem (Figure 1).

Resistance of Enterobacteria to 3rd generation cephalosporins by the production of Extended Spectrum Betalactamase (ESBL) in the isolates was $4 \%$.

All isolates of streptococci and staphylococci were sensitive to penicillins and meticillin, respectively.

All isolated Pseudomonas strains were sensitive to imipenem, fluoroquinolones and gentamicin, and only one was resistant to ceftazidime.

Probabilistic antibiotic therapy initially administered was the combination of amoxicillin-clavulanic acid in addition to gentamicin, except for patients with severe sepsis, who received a triple combination: ceftriaxone + gentamycin + metronidazole. An adaptation of the antibiotherapy was systematically made after the results of the microbiological analysis.

\section{Discussion}

Bacterial peritonitis is associated in developing countries 
with a high risk of mortality. An effective microbiological diagnosis followed by appropriate antibiotic therapy improves the results of the treatment [4].

The microbiology of peritonitis is derived from the intestinal flora [5]. Several studies have confirmed the microbial polymorphism in peritonitis but a pathogenic role is proved for a small number [6].

E. coli is the most common germ, so the consensus of the French Society of anesthesia resuscitation recommends to take into account, during the probabilistic antibiotherapy of community peritonitis, enterobacteria [2].

The French Consensus Conference of 2000 recommended associating amoxicillin-clavulanic acid with an aminoglycoside [2].

In our context, the evolution of the resistance of Enterobacteria, responsible for peritonitis in children, to amoxicillin-clavulanic acid increased between 2006 to 2010 from $16 \%$ to $36 \%$ and it decreased in 2018 to $32 \%$ (figure 2).

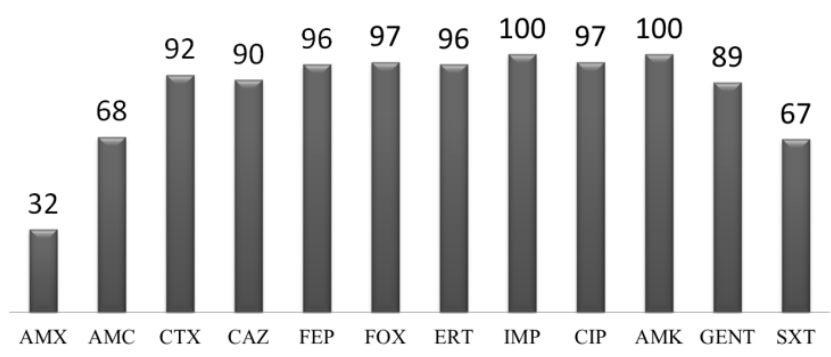

Figure 1. Percentage of susceptibility of Escherichia. coli to antibiotics.

AMX: amoxicillin; AMC: amoxicillin-clavulanic acid; CTX: ceftriaxone; CAZ: ceftazidime; FEP: cefepime; FOX: cefoxitin, CIP: ciprofloxacin; ERT: ertapenem; IMP: imipenem; GENT: gentamicin; AMK: amikacin; SXT: sulfamethoxazole - trimethoprim.

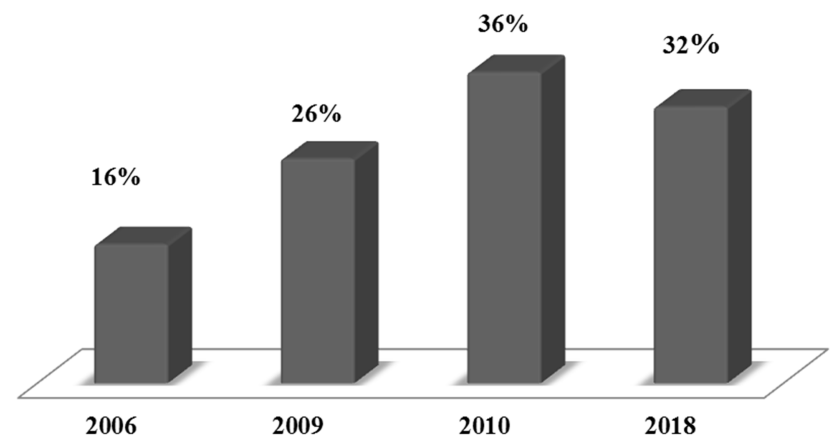

Figure 2. Evolution of resistance of enterobacteria to amoxicillin-clavulanic acid at the University Hospital of Marrakech.

The sensitivity of enterobacteria to the amoxicillinclavulanic acid combination in our study is reduced (68\%) compared to a recently published French study (90.3\%) [7, 8]. This rate was $87 \%$ in another French study [9] and $81 \%$ in a Scandinavian study [10] (Figure 3).

Several studies show an inconsistent action on Gramnegative bacteria and a resistance of more than $30 \%$ of Escherichia coli to clavulanic acid due to the secretion of the enzyme b-lactamase TEM-1 which is not inhibited by this molecule and by the hypersecretion of penicillinases [11].
The percentage of Enterobacteria resistance to 3rd generation cephalosporins in our series increased slightly between 2010 and 2018 , from $7 \%$ to $9 \%$.

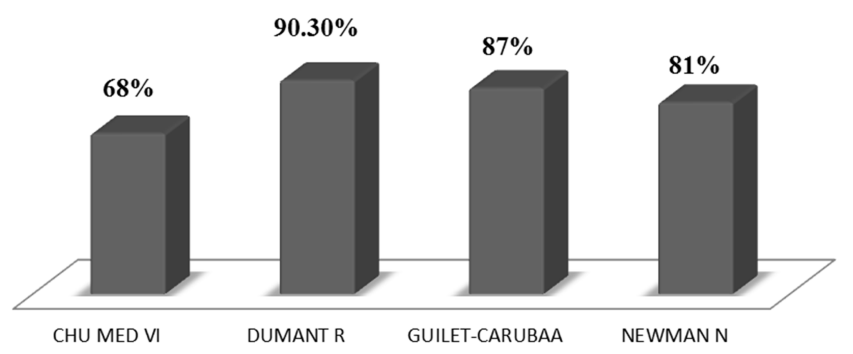

Figure 3. Percentage sensitivity of enterobacteria to amoxicillin-clavulanic acid from other teams.

Globally, the SMART study showed that E. coli is a producer of ESBL in $18 \%$ of cases in 2006-2007, compared to $12 \%$ of cases in 2005 . An important difference between regions was found: the highest frequency of ESBL was in Asia (34.9\%), followed by Latin America (21.6\%), Africa and the Middle East (12.1\%), Europe (8\%) and North America (4.8\%) [12].

The high rate of Pseudomonas in our study can be attributed to repeated antibiotic therapy including selfmedication in our context. Although Pseudomonas was not considered in probabilistic antibiotic therapy, the microbiological results made it possible to include active antibiotics on these germs $[13,18]$.

To treat these infections, antibiotic therapy must be early, with a suitable dosage and covering the bacterial ecology. In view of our findings, a triple combination of ceftriaxone, metronidazole and gentamicin effectively covers $E$. coli and anaerobes $[14,19]$.

But the appearance of resistant strains by the production of ESBL allowed us to reserve this protocol for severe forms of peritonitis, and using amoxicillin-clavulanic acid and gentamicin for simple forms. A monotherapy based on ertapenem is also effective. The piperacillin-tazobactam combination may also be proposed, but it was not systematically studied in our series $[15,17]$.

The use of other antimicrobials as imipenem, cefepime, aztreonam and tigecycline should be limited to prevent the emergence of multiresistant strains [6].

\section{Conclusion}

In light of these data and taking into account the potential gravity of community peritonitis in children, we choose in our context for a triple combination of cefriaxone, metronidazole and gentamicin in severe forms. Special interest must also be paid to reduce the inappropriate use of antimicrobials and to ban self-medication [16]. Further prospective studies should be conducted to follow the evolution of the bacteriological profile of the germs responsible for the peritonitis of the child and to guide probabilistic antibiotherapy. 


\section{Declaration of Interests}

The authors declare no conflicts of interest related to this article.

\section{References}

[1] Bourgoin A, Leone M, Marti C. Prise en charge thérapeutique des péritonites. Med Mal Infect 2004; 34: 183-95.

[2] Société Française d'Anesthésie-Réanimation. Prise en charge des péritonites communautaires - conférence de consensus texte court. Ann Fr Anesth Reanim 2001; 20: 368s-73s.

[3] Nejmi H, Laghla B, Boutbaoucht M, Samkaoui MA. Evolution des résistances de l'Escherichia coli au cours des péritonites communautaires. Med Mal Infect 2011; 41: 21320.

[4] Mahamoudou Sanou, Armand Ky, Caractérisation de la flore bactérienne des péritonites communautaires opérées au Burkina Faso; The Pan African Medical Journal. 2014; 18: 17.

[5] Leone M, Boyadjiev I, Martin C. Péritonites communautaires: quelle antibiothérapie probabiliste? Urg Prat 2007; 80: 31-4.

[6] J. B. Dever, M. Y. Sheikh. Spontaneous bacterial peritonitis bacteriology, diagnosis, treatment, risk factors and prevention. Alimentary pharmacology and therapeutics; volume 41, Issue11, June 2015; 1116-1131.

[7] Montravers P, Lepape A, Dubreuil L, Gauzit R, Pean Y, Benchimol D, Dupont H. Clinical and microbiological profiles of community-acquired and nosocomial intra-abdominal infections: results of the French prospective, observational EBIIA study. J Antimicrob Chemother 2009; 63: 785-94.

[8] Montravers P, Jean-Baptiste S, Tashk P, Péritonites. Le Congrès. Médecins. Conférence d'Essentiel, 2016.

[9] Dumont R, Cinotti R, Lejus C, Caillon J, Boutoille D, Roquilly A, et al. The microbiology of community-acquired peritonitis in children. Pediatr Infect Dis, J 2011; 30: 131-5.

[10] Newman N, Wattad E, Greenberg D, Peled N, Cohen Z, Leibovitz E. Community acquired complicated Intra abdominal infections in children hospitalized during 1995 2004 at a paediatric surgery department. Scand J Infect Dis 2009; 41: 720-6.

[11] Soussy CJ. Comité de l'antibiogramme de la société française de microbiologie. Communiqué 2010 - 2011. Path Biol. 2010; 48: 832-71. PubMed | Google Scholar.

[12] Hawser SP, Bouchillon SK, Hoban DJ, Badal RE. In vitro susceptibilities of aerobic and facultative anaerobic Gram negative bacilli from patients with intra-abdominal infections worldwide from 2005-2007: results from the SMART study. Int J Antimicrob Agents 2009; 34: 585-8.

[13] Profil bactériologique des péritonites communautaires de l'enfant prises en charge au CHU de Marrakech 2012 Published by Elsevier Masson SAS on behalf of the Société francaise d'anesthésie et de réanimation (Sfar).

[14] Nadler EP, Gaines BA, Therapeutic Agents Committee of the Surgical Infection Society. The Surgical Infection Society guidelines on antimicrobial therapy for children with appendicitis. Surg Infect (Larchmt) 2008; 9: 75-83.

[15] Rodriguez-Ban o J, Navarro MD. Extended-spectrum betalactamases in ambulatory care: a clinical perspective. Clin Microbiol Infect 2008; 14 (Suppl. 1): 104-10.

[16] Kilian Friedrich, Simone Nüssle, Tobias Rehlen. Microbiology and resistance in first episodes of spontaneous bacterial peritonitis: implications for management and prognosis, Journal of gastroenterology and hepatology; volume31, Issue6, June 2016 Pages 1191-1195.

[17] W. Zhang, Y. Wu, X. Qi, H. Dai, W. Lu and M. Zhao, "Peritoneal Dialysis-Related Peritonitis with Acinetobacter baumannii: A Review of Seven Cases", Peritoneal Dialysis International, vol. 34, no. 3, pp. 317-321, 2013.

[18] Sartelli M. A focus on intra-abdominal infections. World J Emerg Surg 2010; 5: 2-20.

[19] Ji Young Jang, Seung Hwan Lee, Epidemiology and Microbiology of Secondary Peritonitis Caused by Viscus Perforation: A Single-Center Retrospective Study Surgical Infections; Vol. 16, No. 4; Published Online: 24 Jul 2015. 